\title{
Article \\ Quantitative Evaluation of the Clinical Evolution of Traumatic Onycholysis after Laser Treatment
}

\author{
Felix Marcos-Tejedor ${ }^{1,2, *(\mathbb{D})}$, Raquel Mayordomo ${ }^{3}\left(\mathbb{D}\right.$, Ana María Pérez Pico ${ }^{1}\left(\mathbb{D}\right.$, Pablo A. Cantero-Garlito ${ }^{4}(\mathbb{D}$ \\ and María José Iglesias-Sánchez ${ }^{5}$ \\ 1 Department of Nursing, University Center of Plasencia, University of Extremadura, 10600 Plascencia, Spain; \\ aperpic@unex.es \\ 2 Department of Medical Sciences, Faculty of Health Sciences, University of Castilla-La Mancha, \\ 45600 Talavera de la Reina, Spain \\ 3 Department of Anatomy and Human Embryology, University Center of Plasencia, University of Extremadura, \\ 10600 Plasencia, Spain; rmayordo@unex.es \\ 4 Department of Nursing, Physiotherapy and Occupational Therapy, Faculty of Health Sciences, University of \\ Castilla-La Mancha, 45600 Talavera de la Reina, Spain; pablo.cantero@uclm.es \\ 5 Department of Medical and Surgical Therapy, University Center of Plasencia, University of Extremadura, \\ 10600 Plasencia, Spain; iglesiasmjo@gmail.com \\ * Correspondence: Felix.marcostejedor@uclm.es
}

Citation: Marcos-Tejedor, F.;

Mayordomo, R.; Pérez Pico, A.M.;

Cantero-Garlito, P.A.; Iglesias-

Sánchez, M.J. Quantitative Evaluation of the Clinical Evolution of Traumatic Onycholysis after Laser Treatment. Appl. Sci. 2021, 11, 6817. https:// doi.org/10.3390/app11156817

Academic Editor: Rosario Caltabiano

Received: 12 June 2021

Accepted: 22 July 2021

Published: 24 July 2021

Publisher's Note: MDPI stays neutral with regard to jurisdictional claims in published maps and institutional affiliations.

Copyright: (c) 2021 by the authors. Licensee MDPI, Basel, Switzerland. This article is an open access article distributed under the terms and conditions of the Creative Commons Attribution (CC BY) license (https:/ / creativecommons.org/licenses/by/ $4.0 /)$.

\begin{abstract}
Nail disorders are the most frequent reason for visits to the podiatry clinic. Although they are not a severe health problem, these types of pathologies have a psychosocial impact on patients, affecting their self-esteem and leading to social and professional self-isolation that can cause anxiety and depression. Laser therapy is considered a potential treatment for nail disorders because it is a fast, easily applied treatment that avoids interaction with medication. However, the literature includes no definitive studies with scientific evidence about the efficacy of laser treatment in traumatic onycholysis, the most frequent clinical sign of nail disorders. Objective: We carried out an objective and quantitative study of the efficacy of laser in treating traumatic onycholysis to provide a new, non-invasive treatment for this pathology, and describe an objective method for its clinical evaluation and evolution. Methods: A strictly repetitive protocol of Nd:YAG $1064 \mathrm{~nm}$ laser (PinPointe FootLaser ${ }^{\mathrm{TM}}$, Spain) was applied to nails with idiopathic disorders, following the manufacturer's instructions. The clinical evolution of nail dystrophy was quantitatively evaluated using the affected percentage of the longitudinal axis of the nail. Results: A significant improvement was obtained in nail appearance (dystrophy) in $100 \%$ of cases $(p<0.006)$ after treatment with Nd:YAG $1064 \mathrm{~nm}$ laser. Conclusion: Treating traumatic onycholysis with Nd:YAG $1064 \mathrm{~nm}$ laser can be a good alternative to conventional pharmacological treatments.
\end{abstract}

Keywords: nail disorders; onycholysis; laser; treatment; clinical evaluation

\section{Introduction}

Nail disorders are increasing in prevalence worldwide [1]. These pathologies have a major impact on patient quality of life [2,3], as they can have significant consequences on the physical, emotional [4] and esthetic aspects of people who suffer from them [5]. Psychosocial limitations together with high levels of stigma [6] can also have significant negative effects on the working life and social participation of sufferers who experience these negative effects. These limitations can also occur at any time of life $[7,8]$. Onychodystrophies are one of the most frequent reasons for podiatry visits, and patients demand rapid, easily applied treatments for them $[9,10]$. Comorbidities such as immunodepression status, diabetes mellitus, organ transplant, vascular insufficiency, and the use of corticosteroids and antineoplastic drugs are increasing the prevalence of onychodystrophies and nail infection, and potential for interaction with pharmacological treatment is high $[9,11]$. 
Many pathologic nail abnormalities present onycholysis, including onychodystrophies caused by onychomycosis, psoriasis, lichen planus, traumatisms and the physiological changes of aging [10].

Recent studies have brought advances in laser application for these nail disorders without the use of systemic treatment $[12,13]$ to meet the demand for new and more efficient treatments. Studies in which onychomycosis was treated with $1064 \mathrm{~nm}$ laser reported no definitive evidence to support the efficacy of laser in eliminating infections but concluded that the treatment is innocuous for sufferers and produces clinical improvement in the nails $[13,14]$, despite small structural changes in nail keratin [15]. Four works in the literature describe clinical cases of other nail disorders apart from onychomycosis and nail psoriasis treated with Nd:YAG $1064 \mathrm{~nm}$ laser: one on chronic paronychia, one on median canaliform nail dystrophy, a third on three clinical cases of idiopathic nail disorders, and a fourth on two cases of longitudinal melanonychia. In these studies, the disorders were treated heterogeneously [16-19], with no objective evaluation.

This study reports objective measurement of traumatic onycholysis, one of the main clinical signs of nail disorders, treated homogeneously with Nd:YAG $1064 \mathrm{~nm}$ laser, and describes an objective method for its clinical evaluation.

\section{Materials and Methods}

The study was approved for work with human samples by the Bioethical Commission of the University of Extremadura (ref 35/2012). Eight patients were recruited from the University Podiatry Clinic, and all of them signed a consent form. Patients had none showed clinical signs on their skin or had been diagnosed with a systemic or dermatological disease affecting the nails. The study was performed on 10 nails that after a clinical evaluation were diagnosed with onycholysis on the first toe associated with an anatomical alteration, and fungal infection was ruled out by culture. Clinical signs did not lead to suspicion of the presence of subungual exostosis as the etiology of the onycholysis, and therefore no radiological tests were performed. Nail samples were cultured on a plate with Sabouraud agar and chloramphenicol culture medium, which is selective for the isolation of dermatophyte fungi and yeasts and kept at $30^{\circ} \mathrm{C}$ for three or four weeks. The culture was repeated three times before starting the treatment, to secure the result.

All participants were treated with PinPointe FootLaser ${ }^{\mathrm{TM}}$ (Spain), an Nd:YAG $1064 \mathrm{~nm}$ laser with the following technical specifications: energy per pulse (max) $200 \mu \mathrm{j}$, pulse width (max) $100 \mu$ s and repetition rate $(\max ) 30 \mathrm{~Hz}$. Applying the manufacturer's protocol, participants received three sessions of treatment at monthly intervals. Each session comprised one longitudinal and one transversal pass over the nail and periungual tissue, after debriding the injured part. During each pulse, 10 micro pulses were applied in $0.5 \mathrm{~s}$, with a $0.05 \mathrm{~s}$ interval between micro pulses. This procedure cools the blood capillaries, reducing patient discomfort and minimizing damage to healthy tissue. The application of 10 micro pulses applies $20 \mathrm{~J} / \mathrm{cm} 2$.

To evaluate the efficacy of laser application in treating all nail pathologies and to unify the results, we applied the nail growth theory [20]. In normal conditions, the nail plate can grow from the matrix or nail bed, and its progress is from proximal to distal. Assuming that the cause of the nail disorder ceases, the nail will begin healthy regeneration, and the damaged part will move to distal. Therefore, we evaluated the percentage of the longitudinal axis in which the nail disorder appeared, because according to the nail growth theory, if the cause of the disorder were eliminated, this percentage would decrease (Figure 1). The measurement was performed before treatment and six months post treatment.

The longitudinal axis was measured with the following guidelines. Firstly, the middle point of the nail width at the level of the onychocorneal band and hyponychium was calculated. These two points were combined to define the longitudinal axis of the nail. The total length and the affected part of the longitudinal axis were measured, and the affected percentage of the axis was obtained using the following formula: "affected $\%$ axis $=\mathrm{mm}$ 
affected $\times 100 / \mathrm{mm}$ total axis" (Figure 1). All clinical follow-up was carried out by the same researcher (F.M-T)

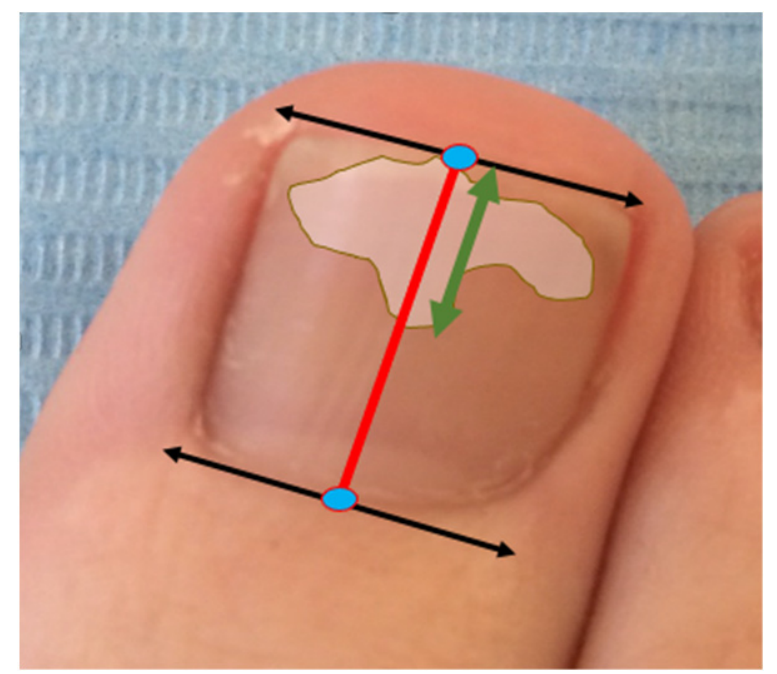

Figure 1. Affected percentage of the longitudinal axis of the nail. The middle point (blue dots), the nail width (black arrow) at the onychocorneal band, and the hyponychium level were calculated. These two points were combined to define the longitudinal axis of the nail (red line). The shaded area simulates the nail lesion. The total length and the affected part of the longitudinal axis were measured (green arrow).

The results were statistically analyzed with SPSS 15.0, applying Student's $t$-test for related samples, with a statistical significance of $5 \%(p<0.05)$.

\section{Results}

Patients had a mean age of $52 \pm 15$ years (range 30-80 years), 6 female and 2 male.

A microbiological culture was performed to rule out fungal infection for all nails analyzed, and the result was negative three times.

To evaluate the affected nails, we used the affected percentage of the axis. Before treatment, the mean percentage of the axis affected was $64.74 \pm 14.92$, and at the six-month post-treatment check-up it was $45.51 \pm 24.12 \%$ (Table 1 and Scheme 1 ). Statistical analysis using the Student's t-test for related samples showed a statistically significant difference $(p<0.006)$. Cohen's $d$ was calculated and obtained a value of 1302, indicating large changes despite the small effect size.

Table 1. Detailed results by participant.

\begin{tabular}{|c|c|c|c|c|c|c|c|}
\hline \multirow{2}{*}{ Subject } & \multirow{2}{*}{ Gender } & \multirow{2}{*}{ Age (Years) } & \multicolumn{2}{|c|}{ Longitudinal Affected \% Axis } & \multirow{2}{*}{ Culture } & \multirow{2}{*}{ Clinical Signs } & \multirow{2}{*}{$\begin{array}{c}\text { Anatomical } \\
\text { Position of the Toe }\end{array}$} \\
\hline & & & Before & After & & & \\
\hline \multirow[b]{2}{*}{1} & \multirow{2}{*}{ Female } & \multirow{2}{*}{61} & 73.33 & 71.43 & Negative & Distal onycholysis & Hallux extensus \\
\hline & & & 67.86 & 60.61 & Negative & Distal onycholysis & Hallux extensus \\
\hline 2 & Female & 50 & 41.94 & 28.00 & Negative & Distal onycholysis & Hallux extensus \\
\hline 3 & Male & 56 & 68.57 & 20.00 & Negative & Lateral onycholysis & Hallux abductus valgus \\
\hline \multirow[b]{2}{*}{4} & \multirow[b]{2}{*}{ Female } & \multirow[b]{2}{*}{80} & 84.00 & 60.61 & Negative & Distal onycholysis & Hallux extensus \\
\hline & & & 65.71 & 57.58 & Negative & Distal onycholysis & Hallux extensus \\
\hline 5 & Female & 44 & 63.16 & 51.35 & Negative & Lateral onycholysis & Hallux abductus valgus \\
\hline 6 & Female & 49 & 66.67 & 21.74 & Negative & Distal onycholysis & Hallux extensus \\
\hline 7 & Male & 46 & 79.55 & 76.47 & Negative & Distal onycholysis & Hallux extensus \\
\hline 8 & Female & 30 & 36.67 & 7.41 & Negative & Distal onycholysis & Hallux extensus \\
\hline \multicolumn{2}{|c|}{ Mean } & 52 & 64.74 & 45.52 & & & \\
\hline
\end{tabular}




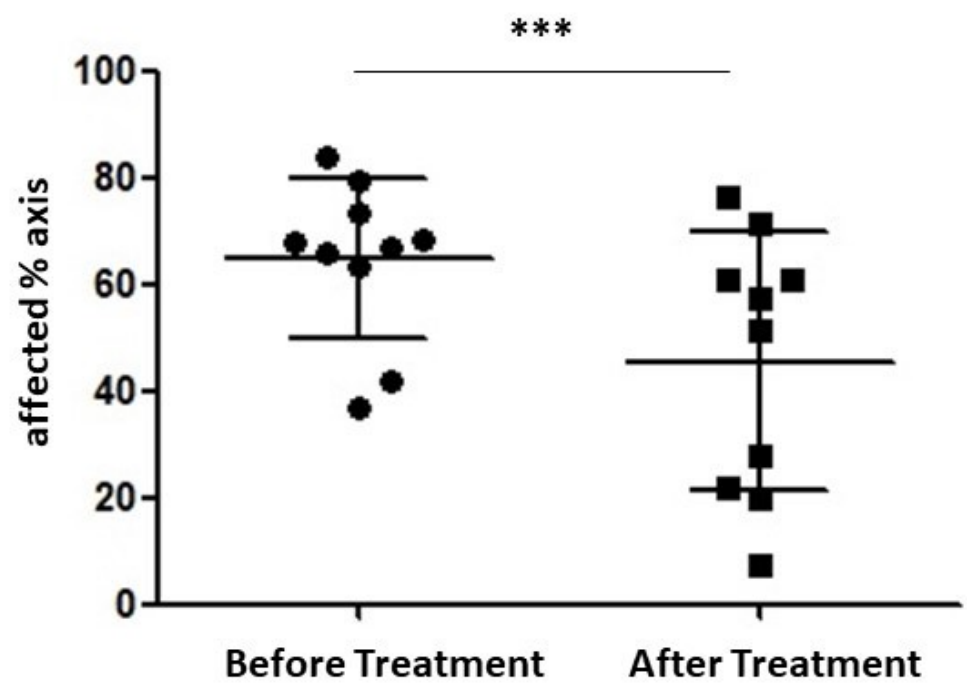

Scheme 1. Change in affected percentage of longitudinal nail axis. $\left({ }^{* *} p<0.006\right)$.

Laser application achieved clinical improvement in $100 \%$ of the nails, although none showed complete remission of symptoms during the study period (Figure 2). No differences were found between the results according to the type of onycholysis and the alteration of the anatomical position of the toe.

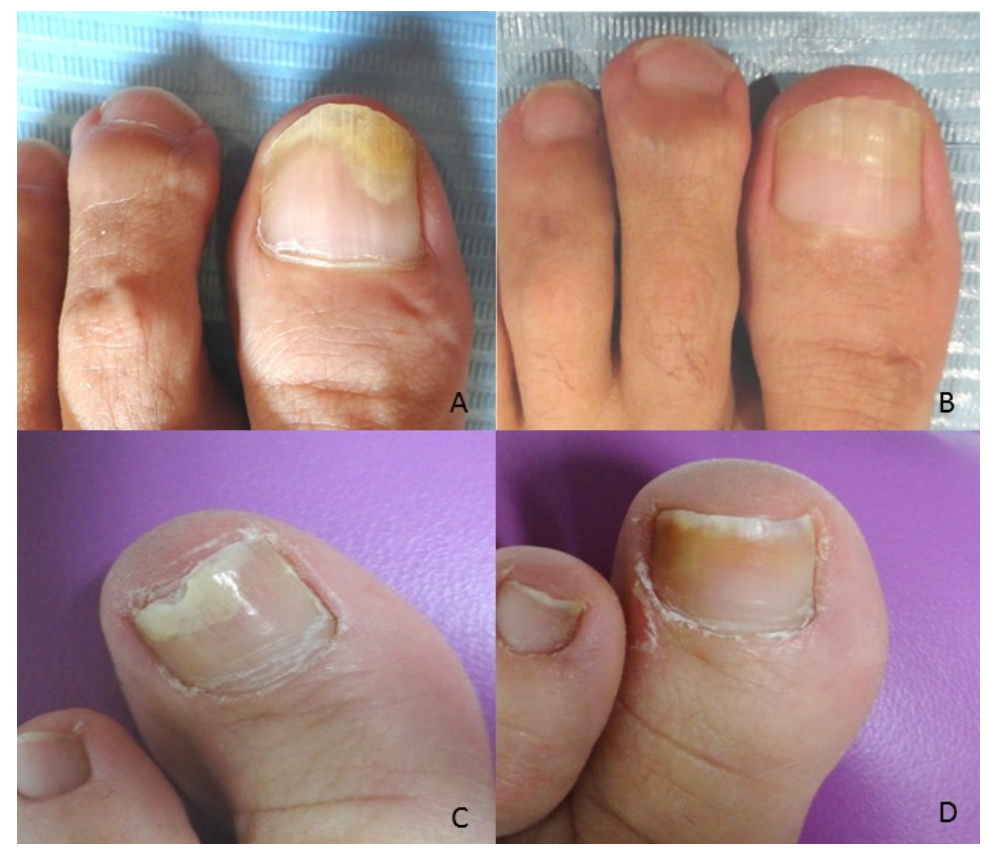

Figure 2. Same patient before and after treatment (A,B). Clinical improvement is observed, with a reduction in the affected area of the longitudinal axis from $68.57 \%$ to $20.00 \%$. Another patient before and after treatment (C,D), showing clinically meaningful changes with a reduction in the affected area of the longitudinal axis from $41.94 \%$ to $28 \%$.

\section{Discussion}

Laser therapy is considered a potential treatment for nail disorders because it is a rapid, easily applied treatment and presents no problems of pharmacological treatments such as side effects or interactions with other drugs [9-11]. The study's principal objective was to measure the efficacy of applying Nd:YAG $1064 \mathrm{~nm}$ laser to treat 10 nails with onycholysis, which were treated homogeneously and clinically evaluated using objective 
criteria. The laser treatment achieved clinical improvement in the nails of all participants, although none showed complete remission of symptoms during the study period.

These findings agree with the results of other new studies contributing to the advancement of laser treatment in nail disorders that concluded there is no scientific evidence about the efficacy of treatment for infections. However, patients experienced evident clinical improvement in their nails [12-19]. The FDA, therefore, does not recommend laser as firstline therapy for onychomycosis [3]. However, Shin et al. (2016) observed structural nail keratin by Raman spectroscopy in three clinical cases. They described structural changes in nail keratin after applying Nd:YAG $1064 \mathrm{~nm}$ laser in onychomycosis treatment [15]. Our results support the idea of laser treatment's beneficial action in the pathologies treated even though they are not due to onychomycosis.

In the literature consulted, we found only descriptions of clinical cases and a pilot study addressing the application of Nd:YAG $1064 \mathrm{~nm}$ laser in disorders other than onychomycosis and psoriasis, such as chronic paronychia, longitudinal melanonychia, and other idiopathic nails disorders [16-19]. The clinical symptoms improved, but the authors concluded that laser could be a nonsurgical alternative with a good aesthetic outcome, and more research is needed on this topic. These works did not treat traumatic onycholysis, each case followed a different protocol, and the clinical evaluation was by clinical observation with no objective measurement method. However, the present work was carried out following the same therapeutic protocol in all cases, photographs were collected of the treated nails showing a qualitative improvement in the appearance of nails with traumatic onycholysis, and improvement was quantified using the method of the affected percentage of the axis.

Statistical analysis confirmed that the improvement observed is objective and significant $(p<0.006)$. Although the rejuvenation or remodeling mechanism of laser action is unclear, we found clinical improvement in $100 \%$ of nails. These results agree with the data in the literature consulted [13,16-19], indicating that the mechanism of laser action should be researched in future studies.

We considered this protocol appropriate because we focused on the progression of the affected area from proximal to distal for the clinical evaluation, based on the nail growth theory [20] and the OSI and NAPSI methods [21,22]. These approaches highlight the importance of evaluating the proximity of the lesion to the nail matrix rather than focusing solely on the affected area. The authors of the NAPSI method proposed a modification after detecting that the nail began healthy regeneration, but the affected area remained unchanged [23]. In this study, the main anatomical landmarks selected to measure the axis (onychocorneal band and hyponychium) prevents changes in the results due to nail trimming if the pathology is located mainly in the distal nail plate.

Further studies should allow a more extended follow-up period until complete renewal of the nail, to entirely rule out recurrence. This study's results were obtained in the short term and a small sample, without considering that follow-up several months posttreatment can show recurrence or complete remission. Additionally, the type of footwear used by the patient and the vascular status patient may also have an influence. It would also be relevant to determine how the use of these new treatments modify patient quality of life.

\section{Conclusions}

We report a quantitative and objective method for clinical evaluation of nail disor-ders, with results showing that applying Nd:YAG $1064 \mathrm{~nm}$ laser to treat traumatic onycholysis led to clinical improvement in all cases analyzed.

Author Contributions: Conceptualization, F.M-T., R.M. and M.J.I.-S.; methodology, F.M.-T.; formal analysis, F.M.-T., A.M.P.P.; investigation, F.M.-T., R.M. and M.J.I.-S.; resources, R.M. and M.J.I.-S.; data curation, F.M-T.; writing-Original draft preparation, F.M.-T. and P.A.C.-G.; writing-Review and editing, F.M.-T., P.A.C.-G., A.M.P.P., R.M. and M.J.I.-S.; supervision, R.M., M.J.I.-S. All authors have read and agreed to the published version of the manuscript. 
Funding: This study was funded by the Regional Government of Extremadura and the European Regional Development Fund (ERDF) through a grant to the research group (code CTS020, reference GR18182).

Institutional Review Board Statement: Not applicable.

Informed Consent Statement: The study was conducted according to the guidelines of the Declaration of Helsinki, and approved by the Bioethical Commission of the University of Extremadura (ref 35/2012).

Conflicts of Interest: The authors declare no conflict of interest.

\section{References}

1. Gupta, A.K.; Mays, R.R. The Impact of Onychomycosis on Quality of Life: A Systematic Review of the Available Literature. Ski. Appendage Disord. 2018, 4, 208-216. [CrossRef]

2. Kayarkatte, M.; Singal, A.; Pandhi, D. Impact of Onychomycosis on the Quality of Life: Dermatology Life Quality Index-Based Cross-Sectional Study. Ski. Appendage Disord. 2020, 6, 115-119. [CrossRef] [PubMed]

3. Leeyaphan, C.; Chayangsu, O.; Bunyaratavej, S.; Kulthanan, K.; Bunyaratavej, S.; Pattanaprichakul, P. Onychomycosis: A study of self-recognition by patients and quality of life. Indian J. Dermatol. Venereol. Leprol. 2015, 81, 270-274. [CrossRef]

4. Malakouti, M.; Brown, G.E.; Leon, A.; Wang, E.; Naegeli, A.N.; Edson-Heredia, E.; Levin, E.; Koo, J.Y.M. The dermatologic intimacy scale: Quantitatively measuring the impact of skin disease on intimacy. J. Dermatol. Treat. 2016, 28, 347-352. [CrossRef] [PubMed]

5. Stewart, C.R.; Algu, L.; Kamran, R.; Leveille, C.F.; Abid, K.; Rae, C.; Lipner, S.R. Effect of onychomycosis and treatment on patient-reported quality-of-life outcomes: A systematic review. J. Am. Acad. Dermatol. 2020, 2, 143. [CrossRef]

6. Alpsoy, E.; Polat, M.; FettahlıoGlu-Karaman, B.; Karadag, A.E.; Kartal-Durmazlar, P.; YalCın, B.; Emre, S.; Didar-Balc1, D.; Bilgic-Temel, A.; Arca, E.; et al. Internalized stigma in psoriasis: A multicenter study. J. Dermatol. 2017, 44, 885-891. [CrossRef]

7. Chernoff, K.A.; Scher, R.K. Nail disorders: Kids are not just little people. Clin. Dermatol. 2016, 34, 736-741. [CrossRef] [PubMed]

8. Smith, R.J.; Rubin, A.I. Pediatric nail disorders: A review. Curr. Opin. Pediatr. 2020, 32, 506-515. [CrossRef]

9. Scher, R.K.; Rich, P.; Elewski, B.; Pariser, D. The epidemiology, etiology, and pathophysiology of onychomycosis. Semin. Cutan. Med. Surg. 2013, 32, S2-S4. [CrossRef]

10. Rich, P.; Elewski, B.; Scher, R.K.; Pariser, D. Diagnosis, Clinical Implications, and Complications of Onychomycosis. Semin. Cutan. Med. Surg. 2013, 32, S5-S8. [CrossRef]

11. Bhatta, A.K.; Huang, X.; Keyal, U.; Zhao, J.J. Laser treatment for onychomycosis: A review. Mycoses 2014, 57, 734-740. [CrossRef]

12. Maranda, E.L.; Nguyen, A.H.; Lim, V.M.; Hafeez, F.; Jimenez, J.J. Laser and light therapies for the treatment of nail psoriasis. J. Eur. Acad. Dermatol. Venereol. 2016, 30, 1278-1284. [CrossRef]

13. Lipner, S.R.; Scher, R.K. Part II: Onychomycosis: Treatment and Prevention of Recurrence. J. Am. Acad. Dermatol. 2019, 80, 853-867. [CrossRef] [PubMed]

14. Yeung, K.; Ortner, V.K.; Martinussen, T.; Paasch, U.; Haedersdal, M. Efficacy of laser treatment for onychomycotic nails: A systematic review and meta-analysis of prospective clinical trials. Lasers Med. Sci. 2019, 34, 1513-1525. [CrossRef]

15. Shin, M.K.; Kim, T.I.; Kim, W.; Park, H.-K.; Kim, K.S. Changes in nail keratin observed by Raman spectroscopy after Nd: YAG laser treatment. Microsc. Res. Tech. 2016, 80, 338-343. [CrossRef] [PubMed]

16. El-Komy, M.H.M.; Samir, N. 1064 Nd: YAG laser for the treatment of chronic paronychia: A pilot study. Lasers Med. Sci. 2013, 30, 1623-1626. [CrossRef] [PubMed]

17. Choi, J.-Y.; Seo, H.-M.; Kim, W.-S. Median canaliform nail dystrophy treated with a $1064 \mathrm{~nm}$ quasi-long pulsed Nd: YAG laser. J. Cosmet. Laser Ther. 2017, 19, 225-226. [CrossRef] [PubMed]

18. Ibrahim, O.; Doumit, J.; Zhang, A. Successful Treatment of Idiopathic Onychodystrophy with 300 Microsecond 1064 nm Nd: YAG Laser. J. Drugs Dermatol. 2015, 14, 750-752.

19. Liu, K.; Tsai, W. Picosecond laser as a promising treatment option for longitudinal melanonychia caused by melanocytic activation: Report of two cases. Photodermatol. Photoimmunol. Photomed. 2019, 36, 163-165. [CrossRef] [PubMed]

20. De Berker, D.A.R.; André, J.; Baran, R. Nail biology and nail science. Int. J. Cosmet. Sci. 2007, 29, 241-275. [CrossRef]

21. Carney, C.; Tosti, A.; Daniel, R.; Scher, R.; Rich, P.; De Coster, J.; Elewski, B. A new classification system for grading the severity of onychomycosis: Onychomycosis Severity Index. Arch. Dermatol. 2011, 147, 1277-1282. [CrossRef] [PubMed]

22. Rich, P.; Scher, R.K. Nail psoriasis severity index: A useful tool for evaluation of nail psoriasis. J. Am. Acad. Dermatol. 2003, 49, 206-212. [CrossRef]

23. Parrish, C.A.; Sobera, J.O.; Elewski, B. Modification of the nail psoriasis severity index. J. Am. Acad. Dermatol. 2005, 53, 745-746. [CrossRef] [PubMed] 